\title{
Impact des pseudo-fèces de moules sur les densités des bactéries hétérotrophes dans le secteur mytilicole de la lagune de Bizerte (Tunisie)
}

\section{Impact of mussel pseudo-feces on heterotrophic bacteria densities in the mussel farm sector of the Bizerte Lagoon (Tunisia)}

\author{
Naceur Essid, Ezzeddine Mahmoudi, Fehmi Boufahja, Mohamed Dellali, \\ Hamouda Beyrem et Patricia Aissa
}

Volume 20, numéro 4, 2007

URI : https://id.erudit.org/iderudit/016912ar

DOI : https://doi.org/10.7202/016912ar

Aller au sommaire du numéro

Éditeur(s)

Université du Québec - INRS-Eau, Terre et Environnement (INRS-ETE)

ISSN

1718-8598 (numérique)

Découvrir la revue

Citer cet article

Essid, N., Mahmoudi, E., Boufahja, F., Dellali, M., Beyrem, H. \& Aissa, P. (2007). Impact des pseudo-fèces de moules sur les densités des bactéries hétérotrophes dans le secteur mytilicole de la lagune de Bizerte (Tunisie). Revue des sciences de l'eau / Journal of Water Science, 20(4), 383-392.

https://doi.org/10.7202/016912ar
Résumé de l'article

Cette étude se propose de caractériser au niveau du secteur mytilicole de la lagune de Bizerte l'impact des pseudo-fèces de moules sur les densités bactériennes, particulièrement celles des Vibrionaceae et des bactéries hétérotrophes. Les prospections ont été réalisées en hiver 2000 et en été 2001 au niveau de 5 stations plus ou moins proches d'une table d'élevage, aussi bien dans la colonne d'eau que dans le sédiment. Sous le parc, les sédiments étant les plus fortement chargés en fraction fine (78-83\%) et en carbone organique total (COT) $(2,5 \%)$, les densités microbiennes étaient élevées $\left(1,82-2,83 \cdot 10^{8}\right.$ UFC/g de sédiment pour les bactéries hétérotrophes et 2,50-17,32 • $10^{2} \mathrm{UFC} / \mathrm{g}$ pour les Vibrionaceae). Une analyse en composantes principales (ACP) montre une étroite relation entre, d'une part, les concentrations bactériennes et, d'autre part, les teneurs en matière organique et plusieurs variables hydrologiques, particulièrement la température, la salinité et l'oxygène dissous. 


\title{
IMPACT DES PSEUDO-FÈCES DE MOULES SUR LES DENSITÉS DES BACTÉRIES HÉTÉROTROPHES DANS LE SECTEUR MYTILICOLE DE LA LAGUNE DE BIZERTE (TUNISIE)
}

Impact of mussel pseudo-feces on heterotrophic bacteria densities in the mussel farm sector of the Bizerte Lagoon (Tunisia)

\author{
Naceur Essid*, Ezzeddine Mahmoudi, Fehmi Boufahja, Mohamed Dellali, \\ Hamouda Beyrem et Patricia Aissa
}

Laboratoire de Biosurveillance de l'Environnement, Faculté des Sciences de Bizerte, 7021 Zarzouna, Bizerte, Tunisie

Reçu le 30 octobre 2005, accepté le 3 avril 2007

\section{RÉSUMÉ}

Cette étude se propose de caractériser au niveau du secteur mytilicole de la lagune de Bizerte l'impact des pseudo-fèces de moules sur les densités bactériennes, particulièrement celles des Vibrionaceae et des bactéries hétérotrophes. Les prospections ont été réalisées en hiver 2000 et en été 2001 au niveau de 5 stations plus ou moins proches d'une table d'élevage, aussi bien dans la colonne d'eau que dans le sédiment. Sous le parc, les sédiments étant les plus fortement chargés en fraction fine (78$83 \%)$ et en carbone organique total (COT) $(2,5 \%)$, les densités microbiennes étaient élevées $\left(1,82-2,83 \bullet 10^{\underline{8}} \mathrm{UFC} / \mathrm{g}\right.$ de sédiment pour les bactéries hétérotrophes et 2,50-17,32 $\bullet 10^{2}$ $\mathrm{UFC} / \mathrm{g}$ pour les Vibrionaceae). Une analyse en composantes principales (ACP) montre une étroite relation entre, d'une part, les concentrations bactériennes et, d'autre part, les teneurs en matière organique et plusieurs variables hydrologiques, particulièrement la température, la salinité et l'oxygène dissous.

Mots clés : Bactéries hétérotrophes, Vibrionaceae, matière organique, lagune de Bizerte, ferme mytilicole.

\section{ABSTRACT}

This study aims to characterize the effect of high organic loads, produced by a mussel farm in the Bizerte Lagoon, on the distribution of bacterial densities of, especially, marine heterotrophic bacteria and Vibrionaceae in the water column and in the sediment. Five sites located along a distance gradient from the mussel breeding ground were surveyed during the winter of 2000 and the summer of 2001. Below the mussel farm, the sediment was characterized by high percentages of fine particles (78-83\%) and total organic carbon (TOC; $2.5 \%$ ). Furthermore, this station exhibited high bacterial densities, especially of heterotrophic flora $\left(1.82-2.83 \bullet 10^{8} \mathrm{UFC} / \mathrm{g}\right)$ and Vibrionaceae (2.50-17.32 $\left.10^{2} \mathrm{UFC/g}\right)$. Principal components analysis revealed a strong relationship among bacterial density, high organic loads and hydrological parameters, particularly temperature, salinity and dissolved oxygen.

Key Words: Heterotrophic bacteria, Vibrionaceae, Organic matter, Bizerte Lagoon, Mussel farms. 


\section{INTRODUCTION}

En Tunisie, la lagune de Bizerte, un écosystème côtier de $150 \mathrm{~km}^{2}$ de superficie et de $12 \mathrm{~m}$ de profondeur maximale, constitue l'un des sites aquacoles les plus importants. Dans ce plan d'eau, la plus ancienne et la plus productive des fermes aquacoles se situe à proximité de la ville de Menzel Jemil. En effet, l'élevage des huîtres y a commencé en 1951 et celui des moules en 1963 (ANPE, 1989) et depuis, la production conchylicole, très variable d'une année à une autre, a été estimée pour la décennie 1989-1998 à 125,3 t/an dont 116,5 t de moules (93\%), 4,6 t/an d'huîtres (4\%) et 4,1 t/an de palourdes (3\%) (DGPA, 1999). Une chute spectaculaire de la production des bivalves a été observée en 1994 (45 tonnes) et en 1997 (65 tonnes), coïncidant avec des conditions sanitaires particulièrement défavorables, à l'origine d'une interdiction prolongée de la commercialisation des coquillages, et ce, en application de la réglementation en vigueur. De plus, les eaux de ruissellement contribuent à enrichir ce plan d'eau en microorganismes susceptibles de s'accumuler dans les fruits de mer. Ainsi, il a été signalé une épidémie de thypho-parathyphoïde faisant suite à l'ingestion de moules crues (GIMAZANE et MEDHIOUB, 1979). Par ailleurs, ZAOUALI (1993) a relevé dans la lagune des problèmes d'eutrophisation et de mortalité massive de bivalves. Certaines Vibrionaceae sont à l'origine de pathologies chez l'homme et les animaux aquatiques: citons les gastro-entérites provoquées par les genres Vibrio et Aeromonas ou la maladie de l'anneau brun causée chez la palourde par Vibrio tapetis (MAES et PAILLARD, 1992). Aux États-Unis, au cours de la période 1983-1992, LIPP et ROSE (1997) ont constaté que Vibrio spp., et au moins dix autres genres de bactéries, ont été à l'origine d'affections contractées par l'ingestion de mollusques et de crustacés. Des travaux de recherches microbiologiques effectués dans la lagune de Bizerte par DELLALI et al. (2000) ont déterminé que les Vibrionaceae sont assez fréquents, particulièrement pendant la saison estivale, au niveau des palourdes $(76,9 \%)$ et secondairement du sédiment proche des palourdes $(70,3 \%)$ et des moules $(57,6 \%)$ de la station de Menzel Jemil, localisée au niveau de la zone des parcs mytilicoles. En 2001, les mêmes auteurs, en essayant d'évaluer la pollution bactérienne au niveau de la lagune de Bizerte, ont conclu que la station de Menzel Jemil était temporairement polluée par les coliformes, les sulfato-réducteurs et les vibrions originaires des rejets d'eaux usées domestiques. Par contre, des valeurs peu élevées en hydrocarbures aromatiques polycycliques (HAP) (MZOUGHI et al., 2002) et en métaux lourds (YOSHIDA et al., 2002) ont été observées au niveau de ce secteur de la lagune de Bizerte. Selon ESSID et AÏSSA (2002), la surcharge organique au niveau des parcs serait en relation avec un envasement de tout le secteur mytilicole. Dans le cadre d'une problématique générale de suivi des paramètres biologiques dans les zones exploitées par l'homme, le présent article se propose d'étudier les interactions entre les paramètres microbiologiques (bactéries hétérotrophes) et la surcharge organique causée par les pseudo-fèces des moules sous l'influence des caractéristiques hydrobiologiques majeures du secteur étudié de la lagune de Bizerte (température, salinité et oxygène dissous).

\section{MATÉRIELS ET MÉTHODES}

Deux campagnes de prélèvement ont été réalisées dans la lagune de Bizerte, respectivement en décembre 2000 et en août 2001, au niveau du secteur mytilicole de Menzel Jemil (Figure 1). Les dosages et les mesures ont été réalisés une seule fois. Le dénombrement des bactéries hétérotrophes a été effectué selon la méthode du nombre le plus probable (NPP) et les résultats ont été exprimés en unités formant colonies (UFC) après calcul de la moyenne pondérée.

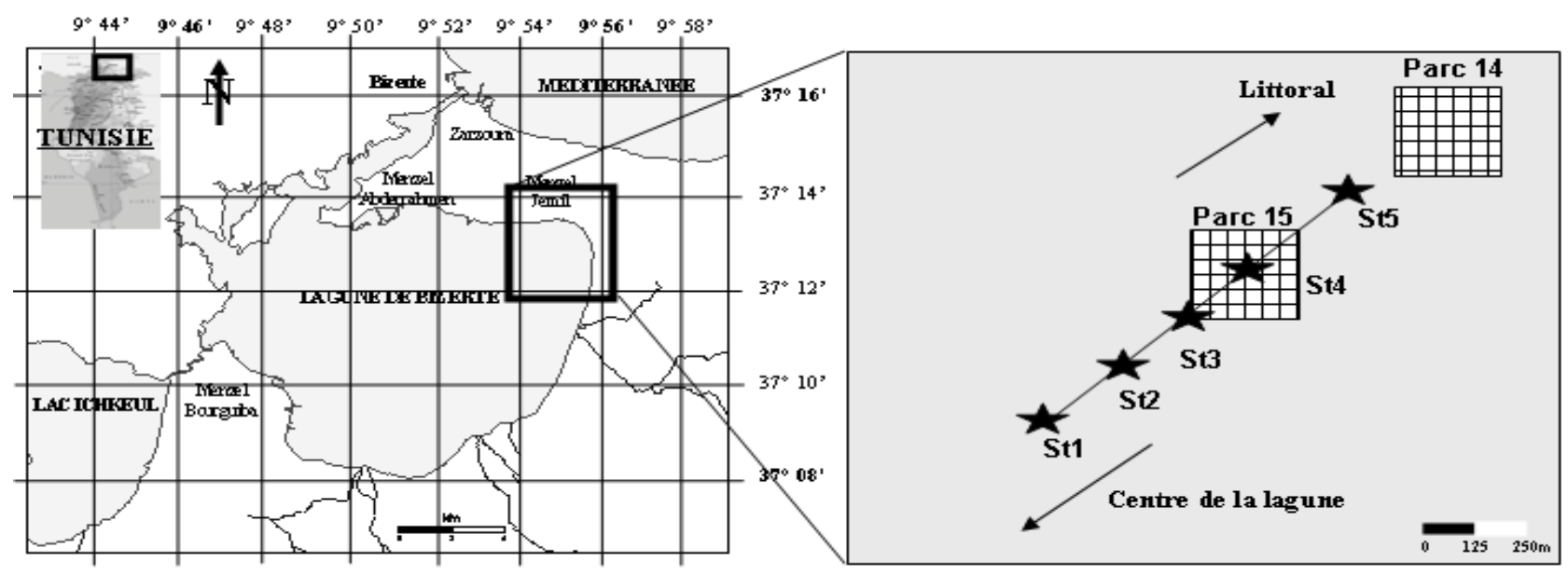

Figure 1. Localisation géographique des 5 stations prospectées au niveau du secteur mytilicole de la lagune de Bizerte au cours des campagnes d'échantillonnage de l'hiver 2000 et de l'été 2001.

Geographic location of the five sampling stations surveyed in the vicinty of the Bizerte Lagoon mussel farm during the winter of 2000 and summer of 2001. 
Le tableau 1 résume les caractéristiques géographiques et bathymétriques des cinq stations prospectées selon une radiale NE-SW passant par une table d'élevage de moules. Tous les paramètres hydrologiques (température, salinité et oxygène dissous) ont été mesurés in situ en surface et au fond de la colonne d'eau. La matière en suspension (MES) est déterminée par filtration des échantillons d'eau prélevés dans des bouteilles en polyéthylène sur des filtres Millipore $(0,45 \mu \mathrm{m})$. Après filtration, les filtres ont été rincés à l'eau douce. Le principe de la détermination de la matière en suspension consiste à peser les filtres avant et après manipulation, la différence des pesées représentant les MES dans le volume d'eau filtrée. Les sédiments récoltés par plongée autonome à l'aide de carottiers en plexiglass ont subi un tamisage humide pour déterminer leur pourcentage en fraction fine (fraction $<63 \mu \mathrm{m}$ ) (WALTER et al., 1997). Sur cette fraction, le carbone organique total (COT) a été dosé par coulométrie à l'aide d'un Coulomat 702 et l'azote total sédimentaire selon la technique Kjeldhal. Les échantillons d'eau et de sédiment destinés à l'étude microbiologique récoltés d'une façon stérile (OMS, 1995), dans des tubes en verre préalablement autoclavés, ont été directement analysés dès le retour au laboratoire. Les bactéries mésophiles hétérotrophes (viables et cultivables à $37^{\circ} \mathrm{C}$ ) (AYSIN et AYTEN, 1998) ont été dénombrées (UFC/unité de volume $(\mathrm{mL})$ ou de masse $(\mathrm{g})$ ) après ensemencement sur gélose nutritive à $2 \%$ de $\mathrm{NaCl}$ par la technique de Plate Counting Agar ou PCA (peptone de caséine, extrait de levure, dextrose, agar). L'isolement des Vibrionaceae (LIPP et ROSE, 1997) et des Aeromonadaceae (GARRITY et al., 2001) a été effectué selon la normalisation française V45-111 (1985) par ensemencement sur milieu sélectif TCBS (thiosulfate - citrate - bile - sodium) (BISIGNANO et al., 1981; BRUNI et al., 1987). Les dénombrements exprimés en UFC sont effectués sur la fraction sédimentaire et au niveau de la colonne d'eau à $20 \mathrm{~cm}$ du fond ou de la surface. Deux logiciels statistiques STATISTICA 6.0 et STATBOX 2.0 ont été utilisés pour la comparaison des moyennes par le test statistique HSD de Tukey, le calcul du coefficient $r$ de Bravais Pearson et une analyse en composantes principales (ACP) appliquée sur les valeurs transformées en $\log (\mathrm{x}+1)$.

\section{RÉSULTATS}

\subsection{Paramètres hydrologiques}

Au niveau du secteur mytilicole de la lagune de Bizerte, les moyennes de la température des eaux pour l'été 2001 $\left(26,76 \pm 1,42{ }^{\circ} \mathrm{C}\right.$ en surface et $26,76 \pm 1,37^{\circ} \mathrm{C}$ au fond) ont doublé $(\mathrm{p}=0,0001)$ par rapport à celles relevées en hiver $2000\left(13,98 \pm 0,54{ }^{\circ} \mathrm{C}\right.$ en surface et $13,58 \pm 0,54^{\circ} \mathrm{C}$ au fond $)$ mais aucune stratification thermique n'a été observée (hiver $\mathrm{p}=0,93$ et été $\mathrm{p}=1$ ). Les salinités dans la colonne d'eau étant relativement homogènes pour chacune des deux saisons considérées, aucune stratification haline (hiver $\mathrm{p}=0,99$ et été $\mathrm{p}=0,96$ ) n'a été relevée (hiver : $37,24 \pm 0,18$ psu en surface et $37,22 \pm 0,11$ psu au fond; été : $39,42 \pm 0,11$ psu en surface et $39,46 \pm 0,09$ psu au fond). L'écart moyen inter-saisonnier des salinités ( $\mathrm{p}=0,0001)$ a été essentiellement lié à l'intensification de l'évaporation et à la rareté des pluies durant l'été. Les teneurs estivales en oxygène dissous au niveau du secteur aquacole $(5,98 \pm 0,45 \mathrm{mg} / \mathrm{L}$ en surface et $6,32 \pm 0,55 \mathrm{mg} / \mathrm{L}$ au fond) ont chuté ( $\mathrm{p}=0,0001)$ par rapport a celles relevées en hiver $(10,23 \pm 0,43 \mathrm{mg} / \mathrm{L}$ en surface et $10,16 \pm 0,21 \mathrm{mg} / \mathrm{L}$ au fond) mais sans qu'apparaisse une stratification des eaux (hiver $\mathrm{p}=0,99$ et été $\mathrm{p}=0,6$ ). Au plan spatial, un déficit en oxygène des eaux du fond a été remarqué sous le parc. Au niveau du secteur d'étude, des pics en MES au fond de la colonne d'eau ont été enregistrés à la station St3 aussi bien en hiver $(27,2 \mathrm{mg} / \mathrm{L})$ qu'en été $(15,8 \mathrm{mg} / \mathrm{L})$. En été, les eaux superficielles de la station St 4 ont été relativement chargées en MES (13,8 mg/L).

\subsection{Paramètres sédimentologiques et géochimiques}

Au cours des deux saisons prospectées, la comparaison statistique des teneurs en fraction fine pour les cinq stations montre que les sédiments de la station St 4 ont été très significativement $(\mathrm{p}<0,01)$ les plus chargés en fraction fine $(80,15 \pm 3,16 \%)$ (Figure 2$)$. Une stabilité temporelle des teneurs élevées en COT au niveau de la station St4 située

Tableau 1. Coordonnées géographiques (latitudes, longitudes) et profondeurs respectives des stations prospectées au niveau du secteur mytilicole de la lagune de Bizerte en hiver 2000 et en été 2001.

Table 1. Geographic data (lattitude, longitude) and depths for the five stations surveyed in the vicinity of the Bizerte Lagoon mussel farm during the winter of 2000 and summer of 2001.

\begin{tabular}{cccccc}
\hline STATION & ST1 & ST2 & ST3 & ST4 & ST5 \\
\hline $\begin{array}{c}\text { LOCALISATION } \\
\text { PAR GPS }\end{array}$ & $\mathrm{N} 37^{\circ} 13^{\prime} 094$ & $\mathrm{~N} 37^{\circ} 13^{\prime} 159$ & $\mathrm{~N} 37^{\circ} 13^{\prime} 167$ & $\mathrm{~N} 37^{\circ} 13^{\prime} 190$ & $\mathrm{~N}^{\prime} 37^{\circ} 13^{\prime} 214$ \\
E09 $54^{\circ} 450$ & $\mathrm{E} 09^{\circ} 54^{\prime} 638$ & $\mathrm{E} 09^{\circ} 54^{\prime} 714$ & $\mathrm{E} 09^{\circ} 54^{\prime} 724$ & $\mathrm{E} 09^{\circ} 54^{\prime} 891$ \\
BATHYMÉTRIE & $5 \mathrm{M}$ & $5 \mathrm{M}$ & $5 \mathrm{M}$ & $5 \mathrm{M}$ & $5 \mathrm{M}$ \\
\hline
\end{tabular}


sous le parc $(\mathrm{p}=0,92)$ a été observée. D'après la comparaison statistique des teneurs spatiales en COT, les sédiments de St 4 se sont distingués de ceux des autres sites qui présentent au contraire des charges en matières organiques carbonées similaires (Figure 2). Une stabilité saisonnière des teneurs sédimentaires en azote total est observée pour les stations St1 $(p=0,08)$, St $3(p=0,13)$ et St4 $(p=0,89)$. L'analyse de la variation spatiale des teneurs moyennes en azote total montre que les sédiments de St 4 présentent des minima aussi bien en hiver $(0,31 \pm 0,08 \%)$ qu'en été $(0,28 \pm 0,02 \%)$. La comparaison des valeurs moyennes en azote par station montre des différences hautement significatives $(\mathrm{p}<0,01)$ entre St 4 et le reste des stations (Figure 3). L'analyse du rapport $\mathrm{C} / \mathrm{N}$ montre une diminution progressive en passant de la table mytilicole (St4) vers l'extérieur du parc (Figure 3).

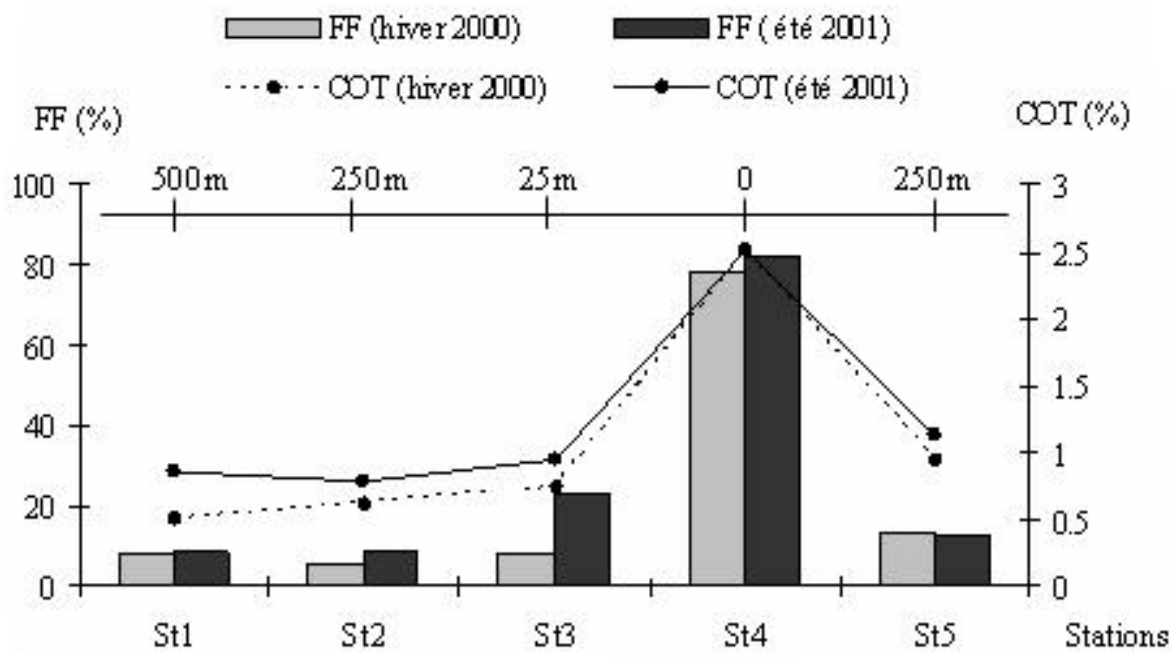

Figure 2. Variation spatiale et saisonnière des teneurs en fraction fine et carbone organique total (COT) des sédiments récoltés aux stations prospectées au niveau de la ferme mytilicole de la lagune de Bizerte en hiver 2000 et en été 2001.

Spatial and seasonal variation of the percent fine fraction and total organic carbon (TOC) in the ssediments collected at five stations surveyed in the vicinity of the Bizerte Lagoon mussel farm during the winter of 2000 and the summer of 2001.

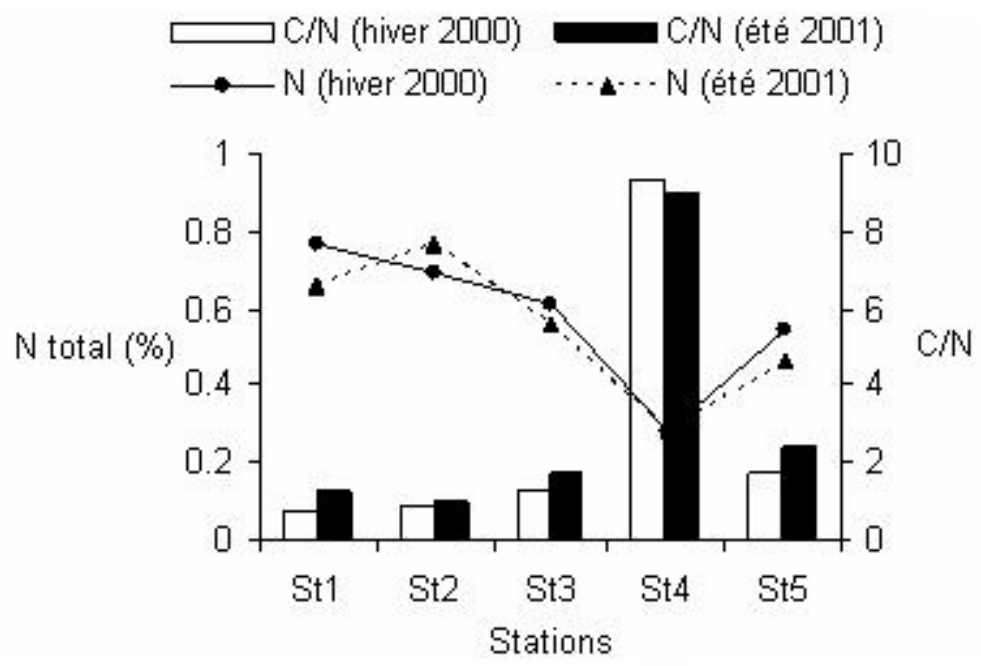

Figure 3. Variation spatiale et saisonnière des teneurs en azote total (\%) et des valeurs du rapport $\mathrm{C} / \mathrm{N}$ des les sédiments récoltés aux cinq stations prospectées au niveau de la ferme mytilicole de la lagune de Bizerte en hiver 2000 et en été 2001.

Spatial and seasonal variation of total nitrogen (\%) and $C / N$ values in the sediments collected at five stations surveyed in the Bizerte Lagoon mussel farm during the winter of 2000 and the summer of 2001. 


\subsection{Paramètres microbiologiques}

L'analyse de la figure 4 montre que les densités des bactéries hétérotrophes des eaux du secteur mytilicole sont nettement plus élevées en hiver. En surface, le rapport densités bactériennes d'hiver/densités bactériennes d'été montre un gradient décroissant avec un maximum de 269 en St 1 et un minimum de 2,8 en St5, alors qu'au fond ce rapport fluctue entre 7,8 (St3 et St5) et 2 en St4. En hiver 2000 (Figure 4), les densités bactériennes les plus élevées pour les eaux de surface ont été observées en dehors du parc à moules (respectivement $4,29 \bullet 10^{6} \mathrm{UFC} / \mathrm{mL}$ en St 1 et $4,03 \cdot 10^{6} \mathrm{UFC} / \mathrm{mL}$ en St5). Le minimum en surface de $1,57 \bullet 10^{6} \mathrm{UFC} / \mathrm{mL}$ a caractérisé les eaux de St3 et St 4 . En comparant les charges hivernales en bactéries hétérotrophes au niveau des eaux de fond, le minimum a été relevé en St $1\left(0,71 \cdot 10^{6} \mathrm{UFC} / \mathrm{mL}\right)$ et le maximum à la station St4 $\left(3,01 \cdot 10^{6} \mathrm{UFC} / \mathrm{mL}\right)$. En été, les eaux de surface de la station St 5 sont apparues les plus chargées en bactéries hétérotrophes $\left(1,46 \bullet 10^{6} \mathrm{UFC} / \mathrm{mL}\right)$ et celles de la station St1 les plus pauvres $\left(0,02 \bullet 10^{6} \mathrm{UFC} / \mathrm{mL}\right)$. Un gradient de charge décroissant est donc observé de St 5 en St1. Pour les eaux de fond, comme en hiver la valeur minimale est toujours relevée en St $1\left(0,15 \bullet 10^{6} \mathrm{UFC} / \mathrm{mL}\right)$ alors que la valeur maximale caractérise St $4 \quad\left(1,44 \bullet 10^{6} \mathrm{UFC} / \mathrm{mL}\right)$.

En hiver 2000, les sédiments les plus chargés en bactéries hétérotrophes ont été ceux de la station St4 (Figure 5) située sous le parc $\left(283,1 \bullet 10^{6} \mathrm{UFC/g}\right)$. Un gradient décroissant de concentration a été observé de la station St4 à la station St1, la plus pauvre en germes $\left(10,7 \cdot 10^{6} \mathrm{UFC} / \mathrm{g}\right)$. En été, les concentrations en germes mésophiles au niveau du sédiment ont été multipliées par 10 (en St5) voire par 20 (St1). Ce sont les sédiments de la station
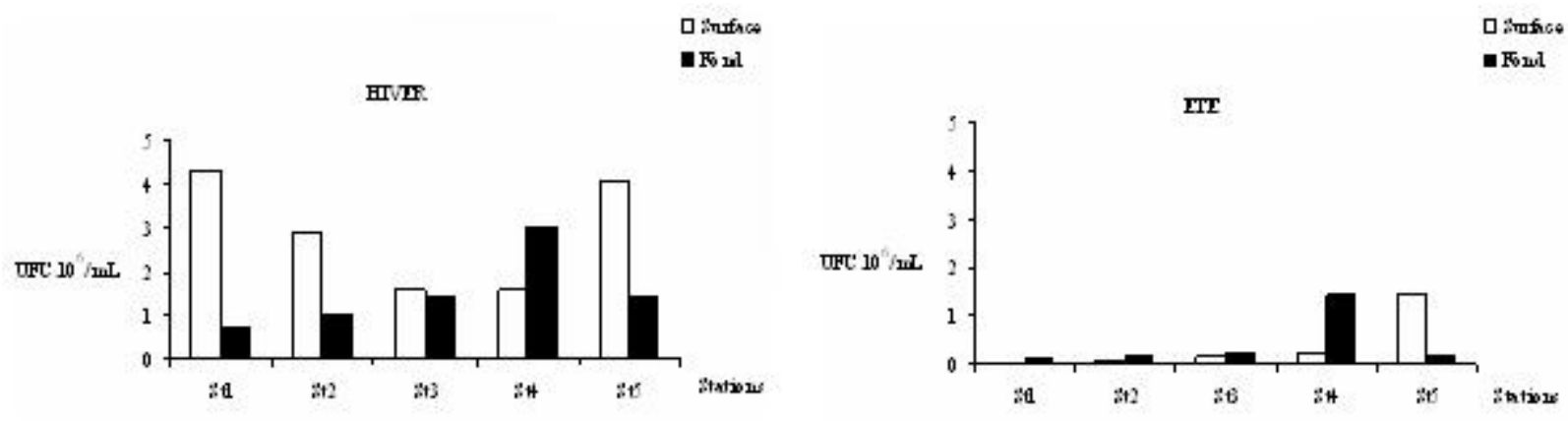

Figure 4. Variation spatiale des concentrations en bactéries hétérotrophes des eaux (UFC.106/ML) des stations prospectées au niveau du parc mytilicole de la lagune de Bizerte en hiver 2000 et en été 2001.

Spatial variation of total heterotrophic bacteria (UFC.106/ML) in the water column of the 5 stations prospected in the mussel farm of Bizerte lagoon during winter 2000 and summer 2001.

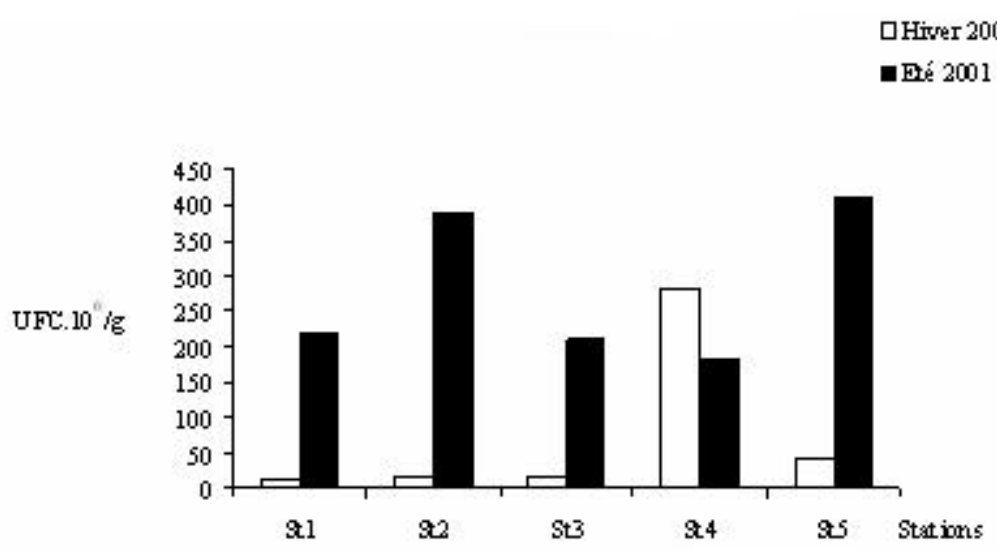

Figure 5. Variation spatiale et saisonnière des teneurs sédimentaires en bactéries hétérotrophes des stations prospectées au niveau du parc mytilicole de la lagune de Bizerte en hiver 2000 et en été 2001. Spatial and seasonal variation of the total sediment content of heterotrophic bacteria at five stations surveyed in the vicinity of the Bizerte Lagoon mussel farm during the winter 2000 and the summer 2001. 
St4 qui ont renfermé, contrairement à l'hiver, un minimum de bactéries hétérotrophes $\left(183 \cdot 10^{6} \mathrm{UFC} / \mathrm{g}\right)$, alors que les valeurs les plus élevées ont caractérisé ceux des stations $\mathrm{St} 5$ $\left(412 \cdot 10^{6} \mathrm{UFC} / \mathrm{g}\right)$ et St2 $\left(389 \cdot 10^{6} \mathrm{UFC} / \mathrm{g}\right)$. En hiver, le minimum en Vibrionaceae a été observé en St3 (200 UFC/g) et le maximum en St5 (401 UFC/g). En été, la concentration la plus élevée a été relevée à la station St 4 (1730 UFC/g) et la plus faible (363 UFC/g) toujours en St3 (Figure 6). Les Vibrionaceae sont assez rares dans les eaux des stations prospectées du secteur mytilicole de la lagune de Bizerte.

D’après la matrice de corrélation établie entre les divers paramètres descripteurs du milieu d'étude, l'oxygène dissous s'est révélé un paramètre limitant l'abondance bactérienne $(\mathrm{r}=+0,91)$, contrairement à la température et la salinité. En ce qui a trait aux sédiments, la teneur en oxygène dissous a été inversement proportionnelle aux charges bactériennes en Vibrionaceae $(\mathrm{r}=-0,78)$ et en bactéries hétérotrophes $(\mathrm{r}=-0,72)$ corrélée à la température et à la salinité $(\mathrm{r}=+0,76)$, à la teneur en fraction fine $(\mathrm{r}=+0,69)$ et en moindre importance aux teneurs en COT $(r=+0,55)$. Les densités sédimentaires en Vibrionaceae ont été significativement corrélées à la fraction fine $(\mathrm{r}=+0,71)$ et marginalement aux teneurs en COT $(r=+0,65)$ et en azote $(r=-0,46)$ (Tableau 2).

\subsection{Analyse en composante principale}

L'Analyse en Composantes Principales (ACP) (Figure 7) a montré que les trois premiers axes factoriels extraient respectivement $53,15 \%, 29,98 \%$ et $11,73 \%$ de la variance totale, soit un pourcentage cumulé de $94,86 \%$. Sur le premier axe F1 extrayant plus de $53 \%$ de l'inertie totale se projette un maximum de variables. Son pôle positif est défini par les teneurs en oxygène dissous des eaux du secteur mytilicole $(\mathrm{CTR}=+0,87)$, alors que sur le côté négatif se projettent la température $(\mathrm{CTR}=-0,87)$, la salinité $(\mathrm{CTR}=-0,89)$, les densités sédimentaires en Vibrionaceae $(\mathrm{CTR}=-0,82)$ et en bactéries hétérotrophes $(\mathrm{CTR}=-0,90)$. L'axe F2 qui extrait environ $30 \%$ de la variance totale est également bipolaire, et il est défini du côté positif par l'azote total sédimentaire $(\mathrm{CTR}=+0,82)$, alors que sur son pôle négatif se projettent les teneurs en fraction fine $(\mathrm{CTR}=-0,76)$ et le rapport $\mathrm{C} / \mathrm{N} \quad(\mathrm{CTR}=-0,82)$. L'axe F3 unipolaire n'est défini que positivement par la teneur des eaux en bactéries hétérotrophes.

\section{DISCUSSION}

Au niveau du secteur mytilicole assez abrité de la lagune de Bizerte, les paramètres hydrobiologiques sont largement influencés par les variations saisonnières des caractéristiques climatologiques de la région, par conséquent une élévation des températures et des salinités et une chute des teneurs en oxygène dissous sont observées en été.

Les sédiments de la station St4 située sous la table mytilicole se sont individualisés des autres sites COT $(2,51 \%)$. Des résultats similaires ont été indiqués dans la littérature par BARRANGUET (1997) (golfe de Fos, France), MIRTO etal. (2000) (Mer Tyrrhénienne, Italie), CHAMBERLAIN et al. (2001) (littoral Irlandais), CRAWFORD et al. (2003) (Tasmanie), CHRISTENSEN

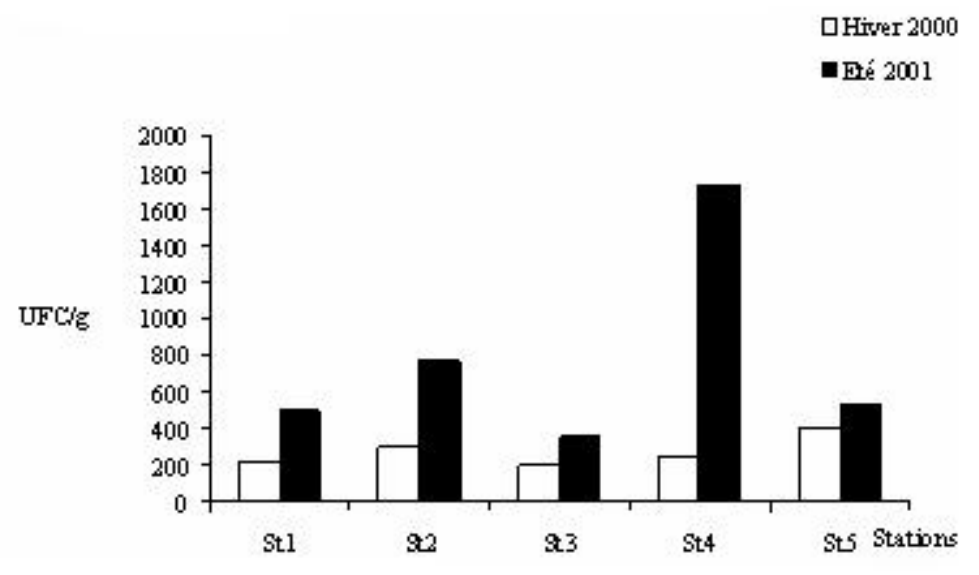

Figure 6. Variation spatiale et saisonnière des teneurs sédimentaires en Vibrionaceae des stations prospectées au niveau du parc mytilicole de la lagune de Bizerte en hiver 2000 et en été 2001.

Spatial and seasonal variation of sediment contente of Vibrionaceae at five stations surveyed in the vicinity of the Bizerte Lagoon mussel farm during the winter of 2000 and the summer of 2001 . 
Tableau 2. Matrice de corrélation des différents paramètres analysés au niveau du secteur mytilicole de la lagune de Bizerte. $\left(\mathrm{T}{ }^{\circ} \mathrm{C}\right.$ : température; $\mathrm{S}$ : salinité; $\mathrm{O}_{2}$ : oxygène dissous; $\mathrm{BHe}$ : bactéries hétérotrophes des eaux; BHs : bactéries hétérotrophes des sédiments; VIBs : Vibrionaceae des sédiments; FF : fraction fine; COT : carbone organique total; $\mathrm{N}$ : azote total; $\mathrm{C} / \mathrm{N}$ : rapport Carbone/Azote. En gras : valeurs de $\mathrm{r}$ significatives à $5 \%$; souligné : valeurs de $\mathrm{r}$ marginalement significatives à $5 \%$.

Table 2: Correlation matrix among the different parameters analysed in the vicinity of the Bizerte Lagoon mussel farm. $\left(T^{\circ} \mathrm{C}\right.$ : temperature; S: salinity; O2:dissolved oxygen ; BHe: total heterotrophic bacteria in water; BHs: total heterotrophic bacteria in sediment; VIBs: sediment Vibrionaceae ; FF: fine fraction ; COT: total organic carbon ; N: total nitrogen ; C/N Carbon/Nitrogen ratio. In bold: $r$ significant at 5\%; underlined: $r$ marginally significant at $5 \%$.

\begin{tabular}{ccccccccccc}
\hline & $\mathbf{T}^{\circ} \mathbf{C}$ & $\mathbf{S}$ & $\mathbf{O}_{2}$ & $\mathbf{B H e}$ & $\mathbf{B H s}$ & VIBs & $\mathbf{F F}$ & $\mathbf{C O T}$ & $\mathbf{N}$ & $\mathbf{C} / \mathbf{N}$ \\
\hline $\mathbf{T}^{\circ} \mathbf{C}$ & 1,00 & & & & & & & & & \\
$\mathbf{S}$ & $\mathbf{0 , 9 9}$ & 1,00 & & & & & & & & \\
$\mathbf{O}_{2}$ & $\mathbf{- 0 , 9 8}$ & $\mathbf{- 0 , 9 9}$ & 1,00 & & & & & & & \\
$\mathbf{B H e}$ & $\mathbf{- 0 , 9 4}$ & $\mathbf{- 0 , 9 1}$ & $\mathbf{0 , 9 1}$ & 1,00 & & & & & & \\
$\mathbf{B H s}$ & $\mathbf{0 , 7 6}$ & $\mathbf{0 , 7 6}$ & $\mathbf{- 0 , 7 2}$ & $\underline{-0,62}$ & 1,00 & & & & & \\
$\mathbf{V I B s}$ & $\mathbf{0 , 8 0}$ & $\mathbf{0 , 7 8}$ & $\mathbf{- 0 , 7 8}$ & $\mathbf{- 0 , 6 7}$ & $\mathbf{0 , 7 3}$ & 1,00 & & & & \\
$\mathbf{F F} \%$ & $\mathbf{0 , 9 1}$ & $\mathbf{0 , 9 1}$ & $\mathbf{- 0 , 9 0}$ & $\mathbf{- 0 , 7 5}$ & $\mathbf{0 , 6 9}$ & $\mathbf{0 , 7 1}$ & 1,00 & & & \\
$\mathbf{C O T} \%$ & $\mathbf{0 , 9 1}$ & $\mathbf{0 , 8 9}$ & $\mathbf{- 0 , 9 2}$ & $-\mathbf{- 0 , 8 6}$ & $\underline{0,55}$ & $\underline{0,65}$ & $\mathbf{0 , 8 6}$ & 1,00 & & \\
$\mathbf{N} \%$ & $\underline{-0,62}$ & $\underline{-0,59}$ & $\underline{0,62}$ & $\underline{0,64}$ & $-0,07$ & $\underline{-0,46}$ & $\underline{-0,51}$ & $-\mathbf{0 , 7 4}$ & 1,00 & \\
$\mathbf{C} / \mathbf{N}$ & 0,39 & 0,31 & $-0,36$ & $\underline{-0,58}$ & 0,14 & 0,11 & 0,10 & $\underline{0,54}$ & $-0,40$ & 1,00 \\
\hline
\end{tabular}

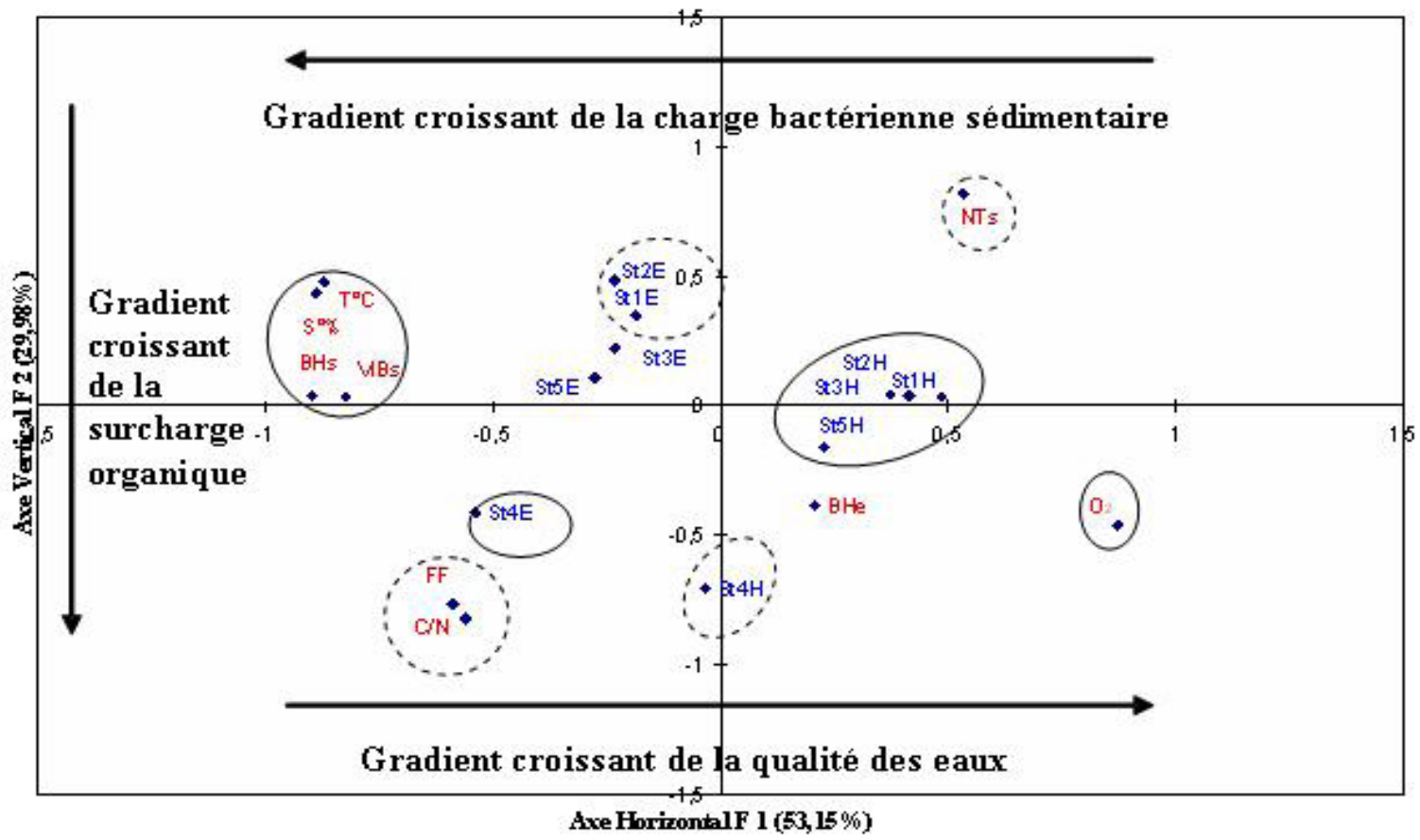

Figure 7: Analyse en Composante Principales effectuée avec les valeurs transformées en $\log (x+1)$ des différents paramètres étudiés au niveau du secteur mytilicole de la lagune de Bizerte en hiver 2000 et été 2001. $\left(\mathrm{T}^{\circ} \mathrm{C}\right.$ : température; $\mathrm{S}:$ salinité; $\mathrm{O} 2$ : oxygène dissous; $\mathrm{BHe}$ : bactéries hétérotrophes des eaux; $\mathrm{BHs}$ : bactéries hétérotrophes des sédiments; VIBs : Vibrionaceae des sédiments; FF : fraction fine; $\mathrm{N}$ : azote total; $\mathrm{C} / \mathrm{N}$ rapport Carbone/Azote.

Principal Compound Analysis realised with $\log (x+1)$ transformed values on the different data studied on mussel farm of Bizerte lagoon during winter 2000 and summer 2001. ( ${ }^{\circ} \mathrm{C}$ : temperature; S: salinity; O2: dissolved oxygen; BHe: water total heterotrophic bacteria; BHs: sediment total heterotrophic bacteria; VIBs: sediment Vibrionaceae; FF: fine fraction; N: total nitrogen; C/N Carbon/Nitrogen ratio. 
et al. (2003) (Nouvelle-Zélande), DANOVARO et al. (2004) (Adriatique, Italie), et HARTSTEIN et STEVENS (2005) (Nouvelle-Zélande). Ces auteurs, particulièrement les italiens qui ont étudié des fermes aquacoles méditerranéennes, affirment que la surcharge en matière organique décomposée au niveau du site aquacole provient essentiellement des fèces et pseudo-feces de moules. CHAMBERLAIN et al. (2001) ajoutent que les particules fines et organiques sédimentées sous les tables peuvent être aussi transportées par le mouvement des masses d'eaux ralenti au niveau du secteur mytilicole. D'autres part, HARTSTEIN et STEVENS (2005) montrent que l'intensité des courants marins est un facteur déterminant la remise en suspension des particules sédimentées sous les tables. Ces derniers ont exigé l'installation de fermes mytilicoles dans des zones non abritées à fort hydrodynamisme pour minimiser l'influence locale des fèces et pseudo-feces de moules et assurer leur meilleure dispersion, ce qui réduit l'enrichissement organique. L'analyse des bactéries hétérotrophes des eaux du secteur étudié a montré une nette fluctuation saisonnière. En hiver, les eaux de fond, et particulièrement celles de surface, sont apparues plus chargées en bactéries, témoignant très probablement d'une intense remise en suspension des particules organiques et sédimentaires riches en bactéries issues essentiellement des sédiments de la station St4. Cette remise en suspension est assurée par l'hydrodynamisme relativement important au niveau de ce secteur nord de la lagune. En effet, des études récentes réalisées en 2002 par l'Institut National des Sciences et Technologies de la Mer (INSTM, 2005) sur la courantologie dans la lagune de Bizerte ont révélé que la circulation des eaux, tant de surface que de fond, suit la direction du vent souvent Nord-Ouest. Toutefois, le déplacement des eaux de fond montre que les zones au nord et au sud de la lagune sont des régions de renouvellement de l'eau. En été, par temps calme et mer de très peu à non agitée, une décantation des particules sédimentaires et organiques provoque l'envasement du secteur mytilicole, ce qui pourrait expliquer la chute des densités bactériennes en ce qui a trait à la colonne d'eau et l'élévation de leurs charges pour ce qui est du sédiment. Les sédiments de la station St 4 ont toujours été très chargés en bactéries mésophiles. En étudiant leurs charges sédimentaires en fonction d'un parc mytilicole et d'une station témoin, MIRTO et al. (2000) ont fait une observation similaire; ceux-ci ont constaté que la densité des bactéries hétérotrophes doublait presque sous le parc, essentiellement à cause de l'abondance de la matière organique de haute qualité offerte par les pseudo-fèces de moules. Selon ces auteurs, cette surcharge organique et la réduction de la pénétration de l'oxygène au niveau des sédiments entraîneraient la réduction de la contribution de la densité des cellules picoeucaryotes. Ces mêmes auteurs résument les changements subits par le peuplement microbien des sédiments des parcs mytilicoles par l'élévation de la densité bactérienne totale, l'augmentation de la contribution des cyanobactéries et de la grande réduction de la contribution des pico-eucaryotes.

En comparaison avec les charges sédimentaires en bactéries mésophiles signalées en Méditerranée (ALBERTELLI et al., 1999; DANOVARO et al., 2000; LA ROSA et al., 2001; MANINI et al., 2003), celles enregistrées en été dans la zone mytilicole de la lagune de Bizerte apparaissent élevées et seulement inférieures à celles relevées à faible profondeur dans des sites souvent confinés, ce qui confirme que notre secteur d'étude subit des contraintes organiques similaires.

Dans les eaux du secteur mytilicole de la lagune de Bizerte, les Vibrionaceae ont été faiblement présentes, ce qui témoigne d'un apport peu important en ces germes par les eaux usées provenant des villes avoisinantes. Pour DELLALI et al. (2000), le phénomène de dilution expliquerait largement leurs faibles densités enregistrées dans l'eau durant la période 1998-1999. Ces auteurs relient les charges maximales en Vibrionaceae relevées en juillet dans l'eau, et au contraire aux mois d'août et de septembre dans les sédiments, à la richesse du milieu en matière organique. Ceci a été vérifié en été 2001 à la station St4, la plus fortement chargée en matière organique provenant des pseudo-fèces de moules. À noter que nos maxima en Vibrionaceae $\left(17,3 \cdot 10^{2} \mathrm{UFC} / \mathrm{g}\right)$ ont été nettement inférieurs à ceux signalés par DELLALI et al. (2001) pour le littoral tout proche mais à quelques centimètres de profondeur $\left(5,98 \cdot 10^{4} \mathrm{UFC} / \mathrm{g}\right)$. Ceci semble confirmer que les apports exogènes en ces germes drainés par les eaux usées des villes avoisinantes sont partiellement limités à la frange littorale, d'une part, et que, d'autre part, les Vibrionaceae dans notre secteur d'étude proviennent essentiellement des parcs enrichis en matière organique par les pseudo-fèces de moules.

L'Analyse en Composantes Principales a confirmé que l'oxygène dissous est un facteur déterminant pour le développement des bactéries du sédiment du secteur des parcs mytilicoles. En effet, le premier axe factoriel F1 semble définir un double gradient (Figure 7) :

- un gradient croissant de la charge bactérienne des stations externes (St1 et St2) vers les stations purement mytilicoles (St4 et St5);

- au contraire, un gradient décroissant de l'oxygénation des eaux de la station St 4 relativement confinée, particulièrement en été vers les stations les plus éloignées de la zone des parcs mytilicoles.

L'axe F2 définit principalement un gradient croissant de la charge en matière organique et en fraction fine, et une vitesse de minéralisation de plus en plus forte en direction de la station St 4 située sous un parc mytilicole. 


\section{CONCLUSION}

Le secteur mytilicole de la lagune de Bizerte est une zone assez abritée et caractérisée par des paramètres hydrologiques largement influencés par des variations climatiques saisonnières. La teneur en oxygène dissous est apparue déterminante pour la distribution spatiale et saisonnière des bactéries originaires du littoral proche et/ou des tables de culture à moules. Celles-ci, hautement productives, affectent localement la qualité des eaux et en particulier celle des sédiments par l'accumulation de matière organique biodégradable. Celle-ci, couplée à un faciès très fin, offre un lieu privilégié pour la multiplication de certaines bactéries chargées de la minéralisation de la matière organique en présence d'oxygène. Les parcs mytilicoles par un rejet continu de pseudo-feces de moules constituent donc un micro-écosystème particulier.

\section{RÉFÉRENCES BIBLIOGRAPHIQUES}

ALBERTELLI, G., A. COVAZZI-HARRIAGUE, R. DANOVARO, M. FABIANO, S. FRASCHETTI et A. PUSCEDDU (1999). Differential responses of bacteria, meiofauna and macrofauna in a shelf area (Ligurian Sea, NW Mediterranean): role of food availability. J. Sea Res., 42, 11-26.

ANPE(AGENCENATIONALE POURLAPROTECTION DE L'ENVIRONNEMENT) (1989). Diagnostic préliminaire pour l'étude de l'équilibre écologique du lac de Bizerte, Projet réalisé par GIC-CNEA-TECI, Tunis, Tunisie, $72 \mathrm{p}$.

AYSIN C. et K. AYTEN (1998). Seasonal distribution of indicator bacteria in seawater from Bosphorus. Rapp. Comm. Int. Mer Mediter., 35, 346-347.

BARRANGUET C. (1997). The role of miocrophytobenthic primary production in a Mediterranean mussel culture. Estuarine Coastal Shelf Sci., 44, 753-765.

BISIGNANO, V., R.B. LOCURTO, T.A. MAUGERI et M. SCOGLIO, 1981. The annual cycle of halophilic vibrios in the brackish lake of Ganzirri. Rev. Int. Oceanogr. Méd., $62,15-21$.

BRUNI V., T. CRISAFI et T. MAUGERI, 1987. Preliminary data on halophilic vibrios in the straits of Messina. Rev. Int. Oceanogr. Méd., 82-83, 67-74.

CHAMBERLAIN J., T. F. FERNANDES, P. READ, T.D. NICKELL et I.M. DAVIES, 2001. Impacts of biodeposits from suspended mussel (Mytilus edulis L.) culture on the surrounding surficial sediments. ICES $J$. Mar. Sci., 58, 411-416.

CHRISTENSEN P. B., R.N. GLUD, T. DALSGAAR et P. GILLESPIE, 2003. Impacts of longline mussel farming on oxygen and nitrogen dynamics and biological communities of coastal sediments. Aquaculture, 218, 567-588.

CRAWFORD C.M., C.K.A. MaCLEOD et I.M. MITCHELL, 2003. Effects of shellfish farming on the benthic environment. Aquaculture, 224, 117-140.

DANOVARO R., D. MARRALE, A. DELLANNO, N. DELLA CROCE, A. TSELEPIDES et M. FABIANO, 2000. Bacterial response to seasonal changes in labile organic matter composition on the continental shelf and bathyal sediments of the Cretan Sea. Prog. Oceanogr., 46, 345-366.

DANOVARO R., C. GAMBI, G.M. LUNA et S. MIRTO, 2004. Sustainable impact of mussel farming in the Adriatic Sea (Mediterranean Sea): evidence from biochemical, microbial and meiofaunal indicators. Mar. Pollut. Bull., 49, 325-333.

DELLALI M., M. EL BOUR et P. AIISSA, 2000. Caractérisation des populations de Vibrionaceae dans la lagune de Bizerte (Tunisie). J. Eur. Hydrol., 31, 91-103.

DELLALI M., M. EL BOUR et P. AÏSSA, 2001. Évaluation de la pollution bactérienne dans la lagune de Bizerte: résultats préliminaires $J$. Res. Océanogr., 26, 18-28.

DGPA, 1999. Annuaires statistiques de la Direction générale de la pêche et de l'aquaculture (DGPA), Tunis, Tunisie,74 p.

ESSID, N. et P. AÏSSA, 2002. Étude quantitative des nématodes libres des secteurs nord et est de la lagune de Bizerte (Tunisie). Bull. Inst. Natl. Sci. Tech. Mer., 29, 5363

GARRITY G.M., M. WINTERS et D.B. SEARLES, (2001). Taxonomic outline of procaryotic Genera Bergey's Manual of Systematic Bacteriology. $2^{\text {nd }}$ Edit. Release 1.0, SPringerVerlag (Éditeur) New York, ÉU, 721 p.

GIMAZANE J.P. et M.N. MADHIOUB (1979). Croissance de l'huître plate dans le lac de Bizerte. Premiers résultats. Bull. Off. Nat. Pêches. 3, 91-94.

HARTSTEIN N.D. et C.L. STEVENS, 2005. Deposition beneath long-line mussel farms. Aquacult. Eng., 33, 192-213.

INSTM. 2005.- INSTITUT NATIONAL DES SCIENCES ET TECHNOLOGIES DE MER. Écologie des lacs BizerteIchkeul, hydrodynamique et ressources aquacoles. Projet El 
Bihera (2002-2006), Rapport 205, Tunis, Tunisie, 60 p.

LA ROSA T., S. MIRTO, A. MAZZOLA et R. DANOVARO, 2001. Differential responses of benthic microbes and meiofauna to fish-farm disturbance in coastal sediments. Environ. Pollut., 112, 427-434.

LIPP E. et J. ROSE, 1997. Le rôle des poissons et des fruits de mer dans les toxi-infections alimentaires aux États-Unis d'Amérique. Rev. Sci. Tech. Off. int. Epiz., 16, 620-640.

MAES P. et C. PAILLARD, 1992. Effet de Vibrio P1, pathogène de Ruditapes philippinarum sur d'autres espèces de bivalves. Dans: Les mollusques marins, biologie et aquaculture, Ifremer, Actes de colloques, 14, Haliotis, France,141-148.

MANINI E., C. FIORDELMONDO, C. GAMBI, A. PUSCEDDU et R. DANOVARO, 2003. Benthic microbial loop functioning in coastal lagoons: a comparative approach. Oceanol. Acta., 26, 27-38.

MIRTO S., T. LA ROSA, R. DANOVARO et A. MAZZOLA, 2000. Microbial and meiofaunal response to intensive Mmussel-farm iodeposition in coastal sediments of the western Mediterranean. Mar. Pollut. Bull., 40, 244-252.

MZOUGHI N., F. HELLAL, M. DACHRAOUI, J-P. VILLENEUVE, C. CATTINI, S. J. DE MORA et A. EL ABED, 2002. Méthodologie de l'extraction des hydrocarbures aromatiques polycycliques. Application à des sédiments de la lagune de Bizerte (Tunisie). C. $R$. Geoscience 334, 893-901.

OMS/PNUE, 1995. Recommandations pour la surveillance sanitaire des zones côtières à usage récréatif et des zones conchylicoles (Partie II \& III). Programme à long terme de surveillance continue et de recherche en matière de pollution de la mer Méditerranée (MED/POL phase II \& III) Danemark, 75 p.

V45-111, 1985. Normalisation française: Produits de pêche, Recherche de Vibrio parahaemolyticus dans les eaux conchylicoles et dans les coquillages marins vivants. Edt AFNOR, Paris, France, 16 p.

WALTER P., H. HARTMUT et M. BERNHARD, 1997. Suspended particulate matter budget for the German Bight, Mar. Pollut. Bull. 34, 398-409.

YOSHIDA M., H. HAMDI, I. ABDULNASSER et N. JEDIDI, 2004. Contamination of potentially toxic elements (PTEs) in Bizerte lagoon bottom sediments, surface sediment and sediment repository. In: Ghrabi A, Yoshida M (eds) Study on Environmental Pollution of Bizerte Lagoon. INRST-JICA Publishers, Tunis, Tunisie, p 31-54.

ZAOUALI J., 1993. Écologie des milieux lagunaires tunisiens. Les populations exploitables aux niveaux halieutique et aquacole. Rapport du plan directeur de l'agriculture en Tunisie, Tunis, Tunisie, $86 \mathrm{p}$. 